\title{
Decreased level of serum carnitine might lead to arteriosclerosis progression via the accumulation of advanced glycation end products in maintenance hemodialysis patients
}

Yumi Kamada ${ }^{1,2}$, Takashi Masuda², Kazuhiko Kotani ${ }^{4}$, Shinya Tanaka², Takeshi Nakamura ${ }^{1,3}$, Nobuaki Hamazaki ${ }^{2}$, Yoko Itoh', Ibuki Moriguchi', Naoyuki Kobayashi ${ }^{1}$, Michihito Okubo ${ }^{1}$, Kazuhiro Takeuchi ${ }^{5}$, Shokichi Naito ${ }^{5}$ and Yasuo Takeuchi ${ }^{5}$

\begin{abstract}
Background: Carnitine is reported to improve insulin resistance and reduce oxidative stress. Hyperglycemia and increased oxidative stress are well known to promote the production of advanced glycation end products (AGEs) that can lead to arteriosclerosis in patients with maintenance hemodialysis (HD). In the present study, we aimed to determine whether decreased level of serum carnitine accelerated arteriosclerosis and to clarify the relationships between carnitine, AGEs, and arteriosclerosis in HD patients.

Methods: We recruited 116 patients (65 men and 51 women, $62 \pm 13$ years) undergoing HD three times a week. We measured pre-HD serum free carnitine prior to the first weekly session. AGE level was quantitatively evaluated by measuring skin autofluorescence (SAF) with AGE reader. Arteriosclerosis was evaluated by measuring carotid intima-media thickness (CIMT). Relationships between free carnitine, SAF, and cIMT were analyzed, and significant limiting factors for arteriosclerosis were identified using univariate and multivariate regression analyses.

Results: Free carnitine ranged from 14.9 to $53.3 \mu \mathrm{mol} / \mathrm{L}$ (mean, $28.6 \pm 8.1 \mu \mathrm{mol} / \mathrm{L}$ ). Free carnitine was negatively correlated with SAF $(r=-0.223, P=0.017)$ and $\mathrm{CIMT}(r=-0.252, P=0.006)$. SAF was positively correlated with CIMT $(r=0.263, P=0.005)$. Free carnitine was identified as a significant independent limiting factor for CIMT $(\beta=-0.194, P=0.037)$.
\end{abstract}

Conclusions: Decreased level of serum carnitine might lead to the progression of arteriosclerosis via the AGE accumulation in HD patients.

Keywords: Advanced glycation end products, Arteriosclerosis, Carnitine, Hemodialysis

\footnotetext{
* Correspondence: tak9999@med.kitasato-u.ac.jp

${ }^{2}$ Department of Angiology and Cardiology, Kitasato University Graduate

School of Medical Sciences, 1-15-1 Kitasato, Minami-ku, Sagamihara,

Kanagawa, Japan

Full list of author information is available at the end of the article
} 


\section{Background}

In maintenance hemodialysis (HD) patients, oxidative stress and hyperglycemia are known to contribute to the formation of advanced glycation end products (AGEs) that are formed by non-enzymatic glycation reactions between reduced sugars and biological macromolecules such as proteins $[1,2]$. In addition, AGEs are slowly metabolized in HD patients, given the role of the kidney in their degradation and excretion [3]. Consequently, AGE accumulation accelerates in the tissues and organs of HD patients [4]. AGEs, binding to AGE receptors on vascular endothelial cells, generate reactive oxygen species and induce vascular inflammation, which can accelerate the progression of arteriosclerosis [5]. Indeed, a number of studies have found that the progression of arteriosclerosis is more accelerated in HD patients due to the high accumulation of AGEs relative to age- and sex-matched healthy individuals [4].

Carnitine is an essential cofactor for the transport of long-chain fatty acids and $\beta$-oxidation in mitochondria [6]. It enhances the oxidative utilization of glucose through the acceleration of pyruvate uptake in mitochondria [7]. Moreover, it improves insulin sensitivity in skeletal muscles by promoting glucose utilization [8]. Carnitine also suppresses oxidative stress by chelating free $\mathrm{Fe}^{2+}$ ion and activating antioxidants [9]. The antioxidant efficacy of carnitine treatment has been reported in various conditions such as hypertension, hypercholesterolemia, atherosclerosis, or aging that increase systemic oxidative stress [10-13]. Thus, carnitine can prevent AGE accumulation in tissues and organs by reducing oxidative stress and improving glucose metabolism. The muscle content of carnitine is substantially reduced in HD patients due to decreased carnitine synthesis in kidney, removal from blood during HD, and insufficient dietary intake, which is reflected in serum carnitine level [14]. Based on this, we hypothesized that decreased level of serum carnitine would enhance AGE accumulation by exacerbating glucose metabolism and increasing oxidative stress, thereby promoting the progression of arteriosclerosis. However, it remains unclear whether decreased level of serum carnitine has this effect in HD patients. To this end, this study aimed to determine whether decreased level of serum carnitine serves as a factor that accelerates arteriosclerosis and to clarify the relationships between carnitine, AGEs, and arteriosclerosis in HD patients.

\section{Methods}

This study was approved by the Bioethics Committee for Clinical Research A of Jichi Medical University Hospital. Written informed consent was obtained from all patients after they received a detailed explanation of the study protocol.
One hundred and thirty patients with end-stage renal disease who received outpatient care for regular maintenance HD three times a week at Sohbudai Nieren Clinic in August 2015 were recruited. Exclusion criteria were as follows: patients with malignancy or dementia or those regularly taking a carnitine-based drug. That is, 8,3 , and 3 patients were excluded for malignancy, dementia, and taking a carnitine-based drug, respectively. Consequently, the final study population consisted of 116 patients on maintenance HD.

\section{Study design}

After clinical characteristics of the patients were obtained from medical records or by interview, we performed blood examinations and AGE measurements and assessed carotid intima-media thickness (cIMT) as an indicator of arteriosclerosis.

\section{Clinical characteristics and blood examination}

The following clinical characteristics were assessed: gender, age, height, dry body weight, body mass index, systolic and diastolic blood pressure, heart rate, duration of $\mathrm{HD}$, prevalence of diabetes mellitus, treatment, and left ventricular ejection fraction. Body mass index was calculated as dry body weight in kilograms divided by height in meters squared. Systolic and diastolic blood pressure and heart rate were measured in the supine position using a vascular profile device (BP-203RPE, Omron Colin, Kyoto, Japan) before the HD session. Left ventricular ejection fraction was measured with an echocardiogram (SSD-5500 system, Aloka, Tokyo, Japan) as an indicator of left ventricular function when patients had stable hemodynamic condition under the optimal dry weight after the HD session.

Blood samples were collected just before the HD session after a 2-day HD interval to measure blood hemoglobin A1c and serum levels of glycated albumin (GA), triglyceride, total cholesterol, low-density lipoprotein cholesterol, and high-density lipoprotein cholesterol as indicators of glycolipid metabolism. We also measured serum levels of albumin, creatinine, uric acid, urea nitrogen, $\beta 2$-microglobumin, C-reactive protein, corrected calcium [15], and phosphate. Serum levels of free carnitine, acyl carnitine, and total carnitine were measured with the enzyme cycling method [16].

Fractional clearance of urea $(\mathrm{Kt} / \mathrm{V})$ was used to evaluate the HD adequacy, which was given by urea clearance $(\mathrm{K}, \mathrm{mL} / \mathrm{min})$, HD treatment time $(\mathrm{t}, \mathrm{min})$, and urea distribution volume $(\mathrm{V}, \mathrm{mL})$ [17].

\section{Advanced glycation end products}

We measured skin autofluorescence (SAF) as an indicator of AGEs. SAF was determined using an AGE reader with an ultraviolet source of specific wavelengths (DiagOptics 
BV, Groningen, The Netherlands), which was reported to reflect AGE accumulation in biopsied skin [18]. The forearm was placed on the AGE reader to measure SAF at three sites, $1 \mathrm{~cm}$ away from each other, where neither bruises nor obvious pigmentation was present. SAF was automatically analyzed with a device connected to a computer that had analysis software installed [19].

\section{Arteriosclerosis}

The carotid intima-media thickness (cIMT) was measured in the supine position with an ultrasonogram (SSD-5500 system, Aloka) by a skilled technician who had no prior knowledge of patients' clinical characteristics. The far and near walls of the right and left common carotid arteries, carotid bulbs, and internal carotid arteries were scanned in both short-axis and long-axis views using a $11-\mathrm{MHz}$ linear probe. The thickest site of the intima-media complex including plaque lesions was measured as the maximum cIMT in increments of $0.1 \mathrm{~mm}$ to assess the severity of arteriosclerosis.

\section{Statistical analysis}

All data are expressed as mean \pm standard deviation. We analyzed relationships between free carnitine, GA, SAF, and cIMT using Spearman's correlation coefficient. $P<0.050$ was considered statistically significant. Univariate and multivariate regression analyses were performed to clarify the relationship between free carnitine and cIMT. In the analyses, we used cIMT as a dependent variable, and factors related to arteriosclerosis progression as independent variables, after assessing the multicollinearity among independent variables [20]. All statistical analyses were performed using the Statistical Package for Social Sciences, version 23.0 for Windows (IBM Corporation, NY, USA).

\section{Results}

Clinical characteristics of the patients are summarized in Table 1. The mean age of patients was $62 \pm$ 13 years, and $54.9 \%$ were male. Patients with a history of type 2 diabetes mellitus comprised $41.4 \%$ of the study population. Serum level of free carnitine ranged from 14.9 to $53.3 \mu \mathrm{mol} / \mathrm{L}$ (mean, $28.6 \pm$ $8.1 \mu \mathrm{mol} / \mathrm{L}$ ), and cIMT from 0.6 to $3.3 \mathrm{~mm}$ (mean, $1.6 \pm 0.6 \mathrm{~mm})$.

Relationships between free carnitine, GA, and SAF are shown in Fig. 1. No significant relationship was found between free carnitine and GA. SAF was negatively correlated with free carnitine $(r=-0.223, P=0.017)$.

Relationships between free carnitine, SAF, and cIMT are shown in Fig. 2. The cIMT was negatively correlated with free carnitine $(r=-0.252, P=0.006)$ and positively correlated with SAF $(r=0.263, P=0.005)$.
Table 1 Clinical characteristics

\begin{tabular}{|c|c|}
\hline Number of patients (male/female) ( $n$ ) & $116(65 / 51)$ \\
\hline Age (years) & $62 \pm 13$ \\
\hline Body mass index $\left(\mathrm{kg} / \mathrm{m}^{2}\right)$ & $21.3 \pm 3.3$ \\
\hline Type 2 diabetes mellitus $(+/-)(n)$ & $48 / 68$ \\
\hline \multicolumn{2}{|l|}{ Treatment (\%) } \\
\hline ARB/ACE inhibitor & 31.0 \\
\hline Iron therapy & 37.1 \\
\hline Systolic blood pressure (mmHg) & $149 \pm 24$ \\
\hline Diastolic blood pressure $(\mathrm{mmHg})$ & $88 \pm 16$ \\
\hline Heart rate (/min) & $77 \pm 12$ \\
\hline Duration of hemodialysis (years) & $7.6 \pm .2$ \\
\hline Kt/N (range) & $1.10 \pm 0.31$ \\
\hline Hemoglobin A1c (\%) & $5.4 \pm 0.7$ \\
\hline Glycated albumin (\%) & $16.7 \pm 3.8$ \\
\hline Triglyceride (mg/dL) & $139 \pm 110$ \\
\hline Total cholesterol (mg/dL) & $169 \pm 32$ \\
\hline Low-density lipoprotein cholesterol (mg/dL) & $94 \pm 26$ \\
\hline High-density lipoprotein cholesterol (mg/dL) & $51 \pm 19$ \\
\hline Albumin (g/dL) & $3.8 \pm 0.3$ \\
\hline Creatinine (mg/dL) & $11.26 \pm 3.08$ \\
\hline Uric acid (mg/dL) & $6.9 \pm 1.4$ \\
\hline Urea nitrogen (mg/dL) & $62.9 \pm 13.5$ \\
\hline Corrected calcium (mg/dL) & $9.0 \pm 0.8$ \\
\hline Phosphate (mg/dL) & $5.7 \pm 1.3$ \\
\hline C-reactive protein (mg/dL) & $0.27 \pm 0.79$ \\
\hline ß2-microglobumin (mg/L) & $24.9 \pm 5.4$ \\
\hline Total carnitine $(\mu \mathrm{mol} / \mathrm{L})$ & $44.5 \pm 11.7$ \\
\hline Free carnitine $(\mu \mathrm{mol} / \mathrm{L})$ & $28.6 \pm 8.1$ \\
\hline Acyl carnitine $(\mu \mathrm{mol} / \mathrm{L})$ & $15.9 \pm 4.7$ \\
\hline Acyl carnitine/free carnitine & $0.6 \pm 0.1$ \\
\hline Skin autofluorescence (arbitrary unit) & $3.4 \pm 0.7$ \\
\hline Left ventricular ejection fraction (\%) & $66.9 \pm 8.9$ \\
\hline
\end{tabular}

Data are presented as mean \pm standard deviation. $\mathrm{Kt} / \mathrm{V}$ is used to evaluate the hemodialysis adequacy

$A R B$ angiotensin II receptor blocker, $A C E$ inhibitor angiotensin-converting enzyme inhibitor

The results of univariate and multivariate regression analyses for cIMT are shown in Table 2. There was no significant multicollinearity in independent variables. Age, prevalence of diabetes mellitus, creatinine, albumin, and free carnitine were significantly correlated with $\operatorname{cIMT}(\beta=$ $0.548, P=0.001 ; \beta=0.225, P=0.015 ; \beta=-0.364, P=0.001$; $\beta=-0.336, P=0.001 ; \beta=-0.252, P=0.006$, respectively). Age, albumin, and free carnitine were identified as significant independent limiting factors for cIMT $(\beta=0.310$, $P=0.006 ; \quad \beta=-0.176, \quad P=0.044 ; \quad \beta=-0.194, \quad P=0.037$, respectively). 


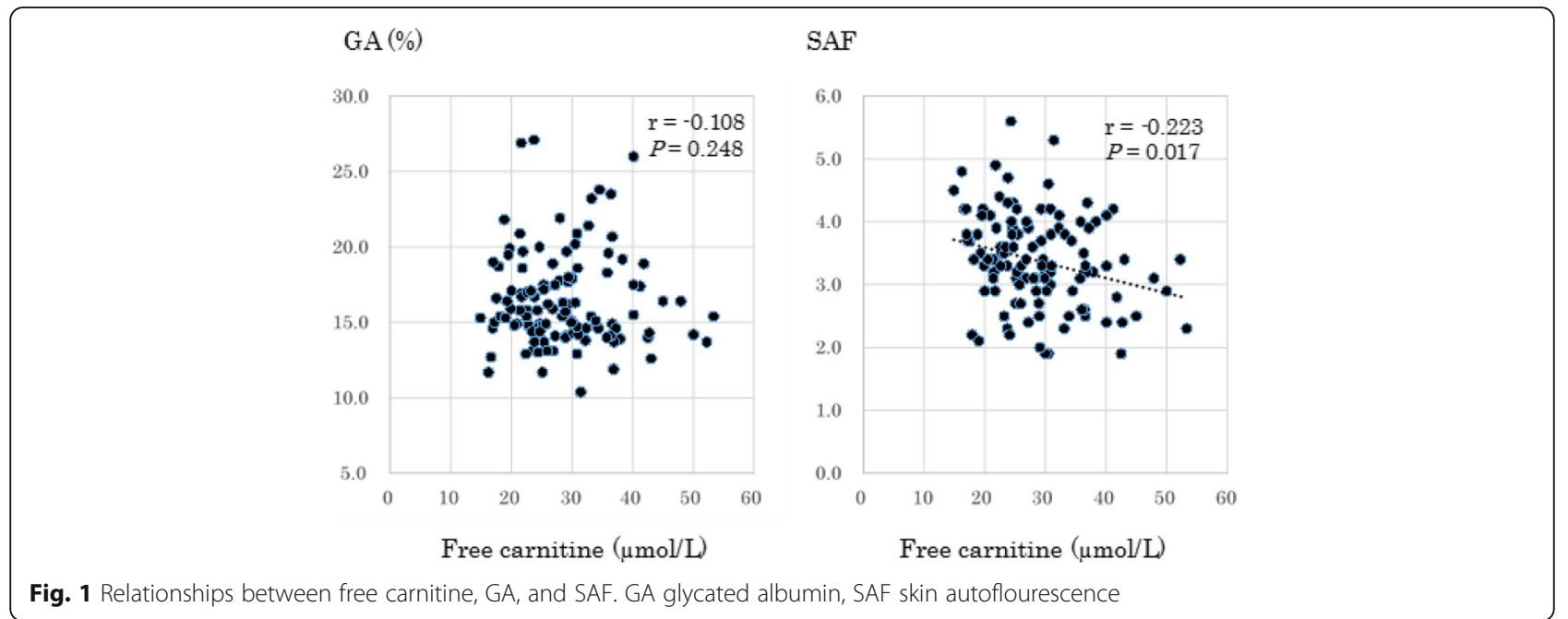

\section{Discussion}

In the present study, we found that a decreased level of serum carnitine was a significant independent determinant of the arteriosclerosis progression in HD patients. That is, multivariate regression analysis revealed that free carnitine was identified as one of significant factors increasing cIMT. In addition, we found that free carnitine was negatively correlated with SAF and that SAF was positively correlated with cIMT.

A previous study showed that decreased serum level of carnitine was independently correlated with increased tissue accumulation of AGEs in HD patients [21]. It has been also reported that AGE accumulation accelerates the progression of arteriosclerosis in HD patients [4]. Therefore, our findings showed that decreased level of serum carnitine might enhance AGE accumulation, thereby promoting the progression of arteriosclerosis in them.

Carnitine has been known to reduce AGE accumulation in tissues and organs by reducing oxidative stress and activating glucose metabolism [22]. However, the present study showed no significant relationship between free carnitine and GA. Because we recruited HD patients whose glucose metabolism was well controlled, most of their GA values were under the target value for glycemic control [23]. That may be the reason why we could not find the significant relationship between them.

Systolic blood pressure and low-density lipoprotein cholesterol are generally known to accelerate arteriosclerosis [24]. However, there were no significant relationships between systolic blood pressure or low-density lipoprotein cholesterol and cIMT in the present study. Savage et al. reported that no significant relationship between systolic blood pressure and cIMT was found in HD patients [25]. As the reason, they stated that the values of systolic blood pressure well controlled with antihypertensives were used for the data analysis. Furthermore, it was reported that hypertension or dyslipidemia was correlated with the progression of arteriosclerosis,

\section{$\operatorname{cIMT}(\mathrm{mm})$}

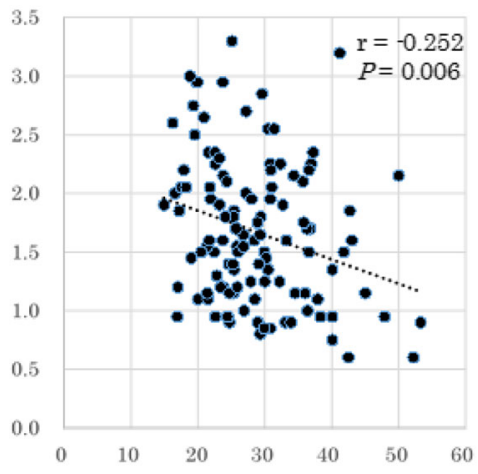

Free Carnitine $(\mu \mathrm{mol} / \mathrm{L})$
$\operatorname{cIMT}(\mathrm{mm})$

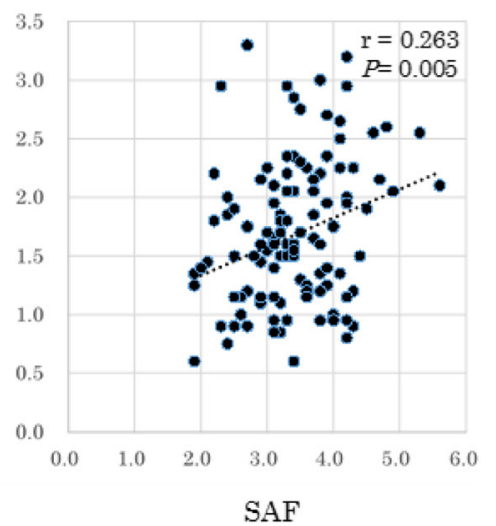

Fig. 2 Relationships between free carnitine, SAF, and cIMT. SAF skin autoflourescence, cIMT carotid intima-media thickness 
Table 2 Results of univariate and multivariate regression analyses for carotid intima-media thickness

\begin{tabular}{|c|c|c|c|c|}
\hline & \multicolumn{2}{|c|}{ Univariate regression } & \multicolumn{2}{|c|}{ Multivariate regression } \\
\hline & $\beta$ & $P$ & $\beta$ & $P$ \\
\hline Gender & 0.132 & 0.158 & 0.159 & 0.108 \\
\hline Age (years) & 0.548 & 0.001 & 0.310 & 0.006 \\
\hline Type 2 diabetes mellitus & 0.225 & 0.015 & 0.106 & 0.235 \\
\hline Body mass index $\left(\mathrm{kg} / \mathrm{m}^{2}\right)$ & -0.162 & 0.082 & -0.037 & 0.685 \\
\hline Systolic blood pressure (mmHg) & -0.089 & 0.344 & -0.065 & 0.432 \\
\hline Low-density lipoprotein cholesterol (mg/dL) & -0.143 & 0.124 & -0.017 & 0.833 \\
\hline Creatinine (mg/dL) & -0.364 & 0.001 & -0.091 & 0.411 \\
\hline Albumin (g/dL) & -0.336 & 0.001 & -0.176 & 0.044 \\
\hline Corrected calcium (mg/dL) & 0.055 & 0.556 & 0.040 & 0.628 \\
\hline Phosphorus (mg/dL) & -0.092 & 0.327 & 0.063 & 0.480 \\
\hline Free carnitine $(\mu \mathrm{mol} / \mathrm{mL})$ & -0.252 & 0.006 & -0.194 & 0.037 \\
\hline
\end{tabular}

when arteriosclerosis was assessed by the mean cIMT that reflected the arterial wall thickness [26]. However, the maximum cIMT was used to assess arteriosclerosis in the present study, which was known to reflect the arterial wall thickness and atheroma formation [26]. The reason why no significant relationships were found between them is to adopt the values of systolic blood pressure and low-density lipoprotein cholesterol that were well controlled with medications and also to adopt the maximum cIMT for data analysis.

There are some limitations worth noting. First, although we speculated that reduced oxidative stress was an underlying mechanism by which carnitine reduces AGEs, we did not evaluate the relationship between carnitine and oxidative stress. In future studies, free radical and lipid peroxide production should be assessed in order to examine the relationship between carnitine and oxidative stress. Second, we could not demonstrate the significant relationship between carnitine and glucose metabolism in the present study. In order to clarify whether carnitine reduces AGEs through the improvement of glucose metabolism, we should perform the reanalysis using large sample sizes in diabetic HD patients and non-diabetic HD patients taking into consideration their condition such as diabetes treatment. Finally, the study design was cross-sectional, and we did not directly examine the effects of carnitine on the progression of arteriosclerosis. Future prospective clinical studies should be planned to elucidate the relationship between carnitine administration, AGEs, and arteriosclerosis.

Because arteriosclerosis is the primary cause of death in HD patients [27], there is a need for promising strategies to prevent its progression. Previous studies showed that carnitine administration attenuated the progression of atherosclerosis and improved cardiovascular dysfunction in HD patients $[28,29]$. Given that serum level of carnitine is significantly reduced in most HD patients
[14], administering carnitine to this population could potentially prevent the progression of arteriosclerosis and improve prognosis.

\section{Conclusions}

Decreased level of serum carnitine might lead to the progression of arteriosclerosis via the AGE accumulation in maintenance HD patients.

\section{Abbreviations}

AGEs: Advanced glycation end products; CIMT: Carotid intima-media thickness; GA: Glycated albumin; HD: Hemodialysis; SAF: Skin autofluorescence

\section{Acknowledgements}

We thank Mr. Makoto Takenaka and Ms. Hitomi Noda for the carotid artery ultrasound examination.

\section{Funding}

This study was not supported by any grants or funding.

\section{Availability of data and materials}

Please contact the corresponding author for data requests.

\section{Authors' contributions}

YK designed the study, collected the clinical data, performed the data analysis, and wrote the manuscript. TM designed the study and wrote the manuscript. KK designed the study and performed the data analysis. TN collected the clinical data. ST, NH, YI, IM, NK, MO, KT, SN, and YT provided substantial revisions to the manuscript. All authors read and approved the final manuscript.

Ethics approval and consent to participate

This study was approved by the Bioethics Committee for Clinical Research A of Jichi Medical University Hospital (RIN No. 13-09).

Consent for publication

Not applicable.

Competing interests

The authors declare that they have no competing interests.

\section{Publisher's Note}

Springer Nature remains neutral with regard to jurisdictional claims in published maps and institutional affiliations. 


\section{Author details}

'Sohbudai Nieren Clinic, Zama, Japan. ${ }^{2}$ Department of Angiology and Cardiology, Kitasato University Graduate School of Medical Sciences, 1-15-1 Kitasato, Minami-ku, Sagamihara, Kanagawa, Japan. ${ }^{3}$ Department of Rehabilitation Sciences, Kitasato University Graduate School of Medical Sciences, Sagamihara, Japan. ${ }^{4}$ Division of Community and Family Medicine, Jichi Medical University, Tochigi, Japan. ${ }^{5}$ Division of Nephrology, Department of Internal Medicine, Kitasato University School of Medicine, Sagamihara, Japan.

Received: 5 August 2016 Accepted: 27 November 2017 Published online: 19 December 2017

\section{References}

1. Miyata T, Wada Y, Cai Z, lida Y, Horie K, Yasuda $Y$, et al. Implication of an increased oxidative stress in the formation of advanced glycation end products in patients with end-stage renal failure. Kidney Int. 1997; 51(4):1170-81.

2. Brownlee $M$, Cerami A, Vlassara $H$. Advanced glycosylation end products in tissue and the biochemical basis of diabetic complications. N Engl J Med. 1988;318(20):1315-21.

3. Miyata T, Ueda Y, Horie K, Nangaku M, Tanaka S, van Ypersele de Strihou C, et al. Renal catabolism of advanced glycation end products: the fate of pentosidine. Kidney Int. 1998;53(2):416-22.

4. Ueno H, Koyama H, Tanaka S, Fukumoto S, Shinohara K, Shoji T, et al. Skin autofluorescence, a marker for advanced glycation end product accumulation, is associated with arterial stiffness in patients with end-stage renal disease. Metabolism. 2008:57(10):1452-7.

5. Basta G, Schmidt AM, De Caterina R. Advanced glycation end products and vascular inflammation: implications for accelerated atherosclerosis in diabetes. Cardiovasc Res. 2004;63(4):582-92.

6. Vaz FM, Wanders RJ. Carnitine biosynthesis in mammals. Biochem J. 2002; 361(3):417-29.

7. Siliprandi N, Di Lisa F, Pieralisi G, Ripari P, Maccari F, Menabo R, et al. Metabolic changes induced by maximal exercise in human subjects following L-carnitine administration. Biochim Biophys Acta. 1990;1034(1):17-21.

8. Rajasekar P, Anuradha CV. L-carnitine inhibits protein glycation in vitro and in vivo: evidence for a role in diabetic management. Acta Diabetol. 2007; 44(2):83-90.

9. Cao Y, Qu HJ, Li P, Wang CB, Wang LX, Han ZW. Single dose administration of L-carnitine improves antioxidant activities in healthy subjects. Tohoku J Exp Med. 2011;224(3):209-13.

10. Sayed-Ahmed MM, Khattab MM, Gad MZ, Mostafa N. L-carnitine prevents the progression of atherosclerotic lesions in hypercholesterolaemic rabbits. Pharmacol Res. 2001;44(3):235-42.

11. Kalaiselvi T, Panneerselvam C. Effect of L-carnitine on the status of lipid peroxidation and antioxidants in aging rats. J Nutr Biochem. 1998;9(10):575-81.

12. Dayanandan A, Kumar $P$, Panneerselvam C. Protective role of $L$-carnitine on liver and heart lipid peroxidation in atherosclerotic rats. J Nutr Biochem. 2001;12(5):254-7.

13. Gomez-Amores $L$, Mate A, Miguel-Carrasco J L, Jimenez $L$, Jos A, Camean $A M$, et al. L-carnitine attenuates oxidative stress in hypertensive rats. J Nutr Biochem. 2007;18(8):533-40.

14. Evans A. Dialysis-related carnitine disorder and levocarnitine pharmacology. Am J Kidney Dis. 2003:41(4 Suppl 4):S13-26.

15. Payne RB, Little AJ, Williams RB, Milner JR. Interpretation of serum calcium in patients with abnormal serum proteins. Br Med J. 1973:4(5893):643-6.

16. Takahashi M, Ueda S, Misaki H, Sugiyama N, Matsumoto K, Matsuo N, et al. Carnitine determination by an enzymatic cycling method with carnitine dehydrogenase. Clin Chem. 1994;40(5):817-21.

17. Gotch FA, Sargent JA. A mechanistic analysis of the National Cooperative Dialysis Study (NCDS). Kidney Int. 1985;28(3):526-34.

18. Meerwaldt R, Hartog JW, Graaff R, Huisman RJ, Links TP, den Hollander NC, et al. Skin autofluorescence, a measure of cumulative metabolic stress and advanced glycation end products, predicts mortality in hemodialysis patients. J Am Soc Nephrol. 2005;16(12):3687-93.

19. Meerwaldt R, Graaff R, Oomen PH, Links TP, Jager JJ, Alderson NL, et al. Simple non-invasive assessment of advanced glycation endproduct accumulation. Diabetologia. 2004;47(7):1324-30.

20. Alain F, ENI Z, Chris S. Elphick: a protocol for data exploration to avoid common statistical problems. Methods Ecol Evol. 2010;1:3-14
21. Adachi T, Fukami K, Yamagishi S, Kaida Y, Ando R, Sakai K, et al. Decreased serum carnitine is independently correlated with increased tissue accumulation levels of advanced glycation end products in haemodialysis patients. Nephrology (Carlton). 2012:17(8):689-94.

22. Fukami K, Yamagishi S, Sakai K, Kaida Y, Adachi T, Ando R, et al. Potential inhibitory effects of L-carnitine supplementation on tissue advanced glycation end products in patients with hemodialysis. Rejuvenation Res. 2013;16(6):460-6.

23. Nakao T, Inaba M, Abe M, Kaizu K, Shima K, Babazono T, et al. Best practice for diabetic patients on hemodialysis 2012. Ther Apher Dial. 2015;19:40-66.

24. Sarnak MJ, Levey AS, Schoolwerth AC, Coresh J, Culleton B, Hamm LL, et al. Kidney disease as a risk factor for development of cardiovascular disease: a statement from the American Heart Association councils on kidney in cardiovascular disease, high blood pressure research, clinical cardiology, and epidemiology and prevention. Hypertension. 2003:42(5):1050-65.

25. Savage T, Clarke AL, Giles M, Tomson CR, Raine AE. Calcified plaque is common in the carotid and femoral arteries of dialysis patients without clinical vascular disease. Nephrol Dial Transplant. 1998;13(8):2004-12.

26. Nakashima A, Yorioka N, Asakimori Y, Ito T, Masaki T, Shigemoto K, et al. Different risk factors for the maximum and the mean carotid intima-media thickness in hemodialysis patients. Intern Med. 2003;42(11):1095-9.

27. Nakai S, Masakane I, Akiba T, Shigematsu T, Yamagata K, Watanabe $Y$, et al. Overview of regular dialysis treatment in Japan as of 31 December 2006. Ther Apher Dial. 2008;12(6):428-56.

28. Higuchi T, Abe M, Yamazaki T, Mizuno M, Okawa E, Ando $H$, et al. Effects of levocarnitine on brachial-ankle pulse wave velocity in hemodialysis patients: a randomized controlled trial. Nutrients. 2014;6(12):5992-6004.

29. van Es A, Henny FC, Kooistra MP, Lobatto S, Scholte HR. Amelioration of cardiac function by L-carnitine administration in patients on haemodialysis. Contrib Nephrol. 1992;98:28-35.

\section{Submit your next manuscript to BioMed Central and we will help you at every step:}

- We accept pre-submission inquiries

- Our selector tool helps you to find the most relevant journal

- We provide round the clock customer support

- Convenient online submission

- Thorough peer review

- Inclusion in PubMed and all major indexing services

- Maximum visibility for your research

Submit your manuscript at www.biomedcentral.com/submit 